doi:10.13108/2021-13-1-31

\title{
JOINT ESTIMATES FOR ZEROS AND TAYLOR COEFFICIENTS OF ENTIRE FUNCTION
}

\section{G.G. BRAICHEV}

\begin{abstract}
In the paper, for an entire function $f(z)=\sum_{n=0}^{\infty} f_{n} z^{n}$, we provide asymptotic and uniform bounds of commensurability of the growth of zeroes and the decaying of the Taylor coefficients one with respect to the other. As an initial point for these studies, the following Hadamard statement serves: if the coefficients of the series obey the inequality $\left|f_{n}\right| \leqslant \varphi(n)$ with some function $\varphi(x)$, then the absolute values of the zeroes grows faster than $1 / \sqrt[n]{\varphi(n)}$. In the present work we improve recently obtained lower bound for the joint growth of the zeroes and the coefficients via the maximal term of the Taylor series of the function $f(z)$ or via the counting function of its zeroes. The employing of Hadamard-rectified coefficients of the series give an opportunity to establish corresponding two-sided estimates. By the methods developing classical ideas we find a numerical dependence of such estimates on the sizes of the gaps of the power series representing the entire function. In particular, we find asymptotic identities relating the zeroes and the coefficients of an entire function. The obtained estimates are sharp and strengthen the known results by other authors.
\end{abstract}

Keywords: Taylor coefficients, Hadamard-rectified zeroes of entire function.

Mathematics Subjects Classifications: 30D20

\section{INTRODUCTION AND REVIEW OF KNOWN RESULTS}

In the paper we consider entire transcendental functions that is, analytic in the entire complex plane functions not coinciding with polynomials. Each such function is represented by its Taylor series

$$
f(z)=\sum_{n=0}^{\infty} f_{n} z^{n}, \quad f_{n}=\frac{f^{(n)}(0)}{n !}, \quad z \in \mathbb{C},
$$

containing infinitely many non-zero terms. We shall use the following characteristics usual in the theory of entire functions:

$$
\begin{aligned}
& M_{f}(r)=\max _{|z|=r}|f(z)| \quad \text { is the maximum of the absolute value of a function, } \\
& \mu_{f}(r)=\max _{n \in \mathbb{N}}\left|f_{n}\right| r^{n} \quad \text { is the maximal term of series (1), } \\
& \nu_{f}(r)=\max \left\{n \in \mathbb{N}_{0}:\left|f_{n}\right| r^{n}=\mu_{f}(r)\right\} \quad \text { is the central index. }
\end{aligned}
$$

Let $E_{0}$ be the class of entire functions possessing infinitely many zeroes. Without loss of generality, we suppose that $f(0) \neq 0$ in series (1). The sequence $\Lambda_{f}=\left\{\lambda_{m}\right\}_{m=1}^{\infty}$ of zeroes of the function $f \in E_{0}$ is written taking the multiplicities in the ascending order of absolute values:

$$
0<\left|\lambda_{1}\right|=\ldots=\left|\lambda_{m_{1}}\right|<\left|\lambda_{m_{1}+1}\right|=\ldots=\left|\lambda_{m_{2}}\right|<\ldots,
$$

G.G. Braichev, Joint estimates of zeros and Taylor coefficients of entire function.

(C) Braichev G.G. 2021.

The reported study was funded by RFBR according to the research project no. 18-01-00236.

Submitted November 15, 2020. 
while the counting and averaged counting functions of the zeroes are denoted by

$$
n_{f}(r)=\max \left\{m \in \mathbb{N}:\left|\lambda_{m}\right| \leqslant r\right\} \quad \text { and } \quad N_{f}(r)=\int_{0}^{r} \frac{n_{f}(t)}{t} d t
$$

respectively.

There are a lot of works devoted to studying the dependence of the growth of an entire function on the decay rate of its Taylor coefficients or on the growth and distribution of its zeroes on the complex plane. In most part of works, such characteristics of the growth of $\ln M_{f}(r)$ as order, type and others are calculated by the coefficients of series (1), see [1]-[9]. In other works, a relative growth of $\ln M_{f}(r)$ and of the counting function $n_{f}(r)$ is studied; a wide list of references can be found in work by Valiron [10]. However, just few attention was paid on studying direct relations between the Taylor coefficients and the zeroes of an entire function, although such relations is needed in special issues of the spectral theory of operators and interpolation problems, where the spectrum or the intepolation nodes are zeroes of some entire function and vice versa. Direct formulae expressing the zeroes via the coefficients and vice versa are too bulky and provide no information on mutual behavior of these sequences [11], [12. This difficulty was overcome by Hadamard [13], [14] and he established that the absolute values of the zeroes $\lambda_{n}$ of a function $f(z)$ grow faster that $\left|f_{n}\right|^{-\frac{1}{n}}$. He proposed a method allowing one to compare relative behavior of Taylor coefficients and the zeroes. The approach was extended by Borel [15], while Valiron transformed the estimates into a sharper form. Let us provide one of the results by Valiron, see [6].

If the coefficients of series (1) satisfy the condition

$$
\frac{f_{n-1} f_{n+1}}{f_{n}^{2}} \rightarrow 0, \quad n \rightarrow \infty,
$$

then the following formulae relating the zeroes and the coefficients hold:

$$
\begin{array}{lll}
f_{n} \sim \frac{(-1)^{n} f_{0}}{\lambda_{1} \lambda_{2} \cdots \lambda_{n}}, & & n \rightarrow \infty, \\
\lambda_{n} \sim-\frac{f_{n-1}}{f_{n}}, & & n \rightarrow \infty .
\end{array}
$$

It should be said that the functions with the coefficients obeying condition (3) have a slow growth, namely, they satisfy the condition

$$
\lim _{r \rightarrow+\infty} \frac{\ln M_{f}(r)}{\ln ^{2} r}=0 .
$$

In his Habilitation thesis, Oskolkov [16] has proved a following statement while he studied the Newton interpolation problem for fast growing nodes.

If the zeroes $\left\{\lambda_{n}\right\}$ of the function $f(z)$ obey the condition

$$
\frac{\ln n}{\left|\lambda_{n}\right|} \searrow 0, \quad n \rightarrow \infty,
$$

then the coefficients of the Taylor series of this function satisfy the condition

$$
\varlimsup_{n \rightarrow \infty} \sqrt[n]{\left|f_{n} \lambda_{1} \lambda_{2} \cdots \lambda_{n}\right|}=1
$$

Let us also provide in our terms some results obtained recently by Ukrainian mathematicians. Pel'chars'ka and Sheremeta [17] proved the following. If $f \in E_{0}$, then

$$
\varlimsup_{n \rightarrow \infty}\left|\lambda_{n}\right| \sqrt[n]{\left|f_{n}\right|} \geqslant 1
$$


There exists $f \in E_{0}$ such that

$$
\varlimsup_{n \rightarrow \infty}\left|\lambda_{n}\right| \sqrt[n]{\left|f_{n}\right|}=1
$$

In [18], Andrusyak obtained the following result. If $f \in E_{0}$, then

$$
\varlimsup_{n \rightarrow \infty}\left|\lambda_{n}\right| \sqrt[n]{\left|f_{n}\right|} \geqslant e^{\lim _{r \rightarrow+\infty} \frac{\ln \mu_{f}(r)}{\nu_{f}(r)} .}
$$

There exists a function in the class $E_{0}$, at which the identity is attained in (8).

Andrusyak and Filevich proved the following result [19]. If $f \in E_{0}$, then

$$
\varlimsup_{n \rightarrow \infty}\left|\lambda_{n}\right| \sqrt[n]{\left|f_{n}\right|} \geqslant e^{\lim _{r \rightarrow+\infty} \frac{N_{f}(r)}{n_{f}(r)}} .
$$

There exists a function in the class $E_{0}$, at which the identity is attained in (9)1.

We are going to formulate a result of a non-asymptotic nature obtained in 1938 by Ostrovski [20]; the notations are introduced below in Section 2.

Let $p \in \mathbb{N}$. If an entire function possesses at least $p$ zeroes and $\left|\lambda_{p}\right| \leqslant R_{p}$, then

$$
\prod_{n=1}^{p}\left(1-\frac{\left|\lambda_{n}\right|}{R_{p}}\right)<\frac{1}{2}, \quad\left|\lambda_{p}\right|>\left(1-\left(\frac{1}{2}\right)^{\frac{1}{p}}\right) R_{p}
$$

here the second inequality follows the first inequality. The constants can not be improved for each $p$.

The aim of the present work is to prove sharper lower bounds, which will imply all aforementioned results. Moreover, we shall obtain two-sided estimates for the zeroes of the function in terms of its Taylor coefficients extended some results by Valiron [6] on lacunary series. The possibility of such extension was hinted by Hadamard [13. In particular, we show that for each entire function $f \in E_{0}$ the Hadamard-rectified coefficients $F_{n}$ of series (1) (see a definition below) satisfy the inequality:

$$
\varliminf_{n \rightarrow \infty} \sqrt[n]{F_{n}\left|\lambda_{1} \lambda_{2} \cdots \lambda_{n}\right|} \geqslant 1
$$

as well as an inequality strengthening (9):

$$
\varliminf_{n \rightarrow \infty} F_{n} \sqrt[n]{\left|\lambda_{n}\right|} \geqslant e^{r \stackrel{\lim _{\rightarrow+\infty}}{\frac{N_{f}(r)}{n_{f}(r)}} .}
$$

We proceed to the main part of the work.

\section{PRELIMINARIES}

Let a function $f$ be defined by series (1), that is,

$$
f(z)=\sum_{n=0}^{\infty} f_{n} z^{n}, \quad f(0)=f_{0}=1, \quad z \in \mathbb{C},
$$

the value $f(0)=1$ is chosen for the convenience. Let $y=G(x)$ be the equation of the NewtonHadamard polygon, that is, the equation of the boundary of the convex hull of the points $\left(n,-\ln \left|f_{n}\right|\right), n \in \mathbb{N}_{0}=\mathbb{N} \cup\{0\}$. This polygon consists of straight segments connecting its vertices $\left(n_{k},-\ln \left|f_{n_{k}}\right|\right), k \in \mathbb{N}_{0}$. Following Valiron, the abscissas of the vertices $n_{k}, k \in \mathbb{N}_{0}$, are called central indices of $f$, more precisely, of the Taylor series of the function $f$. We denote:

$$
F_{n}=e^{-G(n)}, \quad n \in \mathbb{N}_{0} \quad \text { and } \quad R_{0}=1, \quad R_{n}=e^{G(n)-G(n-1)}, \quad n \in \mathbb{N} .
$$

\footnotetext{
${ }^{1}$ In works [17]-19] instead of the zeroes of the function $f(z)$, its $a$-points were considered, that is, the zeroes of the function $f(z)-a$.
} 
We obviously have:

$$
R_{n}=\frac{F_{n-1}}{F_{n}} \quad \text { and } \quad F_{n}=\frac{1}{R_{1} R_{2} \cdots R_{n}}, \quad n \in \mathbb{N}
$$

Let us mention some known properties of the Newton-Hadamard polyline, see, for instance, [7]. The relations hold:

$$
\left|f_{n}\right| \leqslant F_{n}, \quad n \in \mathbb{N}_{0} \quad \text { and } \quad\left|f_{n_{k}}\right|=F_{n_{k}}, \quad k \in \mathbb{N}_{0}
$$

The functions $f(z)=\sum_{n=0}^{\infty} f_{n} z^{n}$ and $F(z)=\sum_{n=0}^{\infty} F_{n} z^{n}$ possess the same maximal terms and central indices:

$$
\begin{aligned}
& \mu_{f}(r)=\max _{n \in \mathbb{N}_{0}}\left|f_{n}\right| r^{n}=\max _{n \in \mathbb{N}_{0}} F_{n} r^{n}=\mu_{F}(r), \\
& \nu_{f}(r)=\max \left\{n \in \mathbb{N}_{0}:\left|f_{n}\right| r^{n}=\mu_{f}(r)\right\}=\max \left\{n \in \mathbb{N}_{0}: F_{n} r^{n}=\mu_{F}(r)\right\}=\nu_{F}(r),
\end{aligned}
$$

and the relations hold:

$$
\left|f_{n}\right| \leqslant \min _{n \in \mathbb{N}_{0}} \frac{\mu_{f}(r)}{r^{n}}=F_{n}, \quad n \in \mathbb{N}_{0} .
$$

The coefficients $F_{n}$ are called Hadamard-rectified coefficients of the function $f(z)$. A piece-wise linearity of $G(x)$ implies the relations:

$$
\begin{aligned}
& \nu_{f}(r)=0, \quad r \in\left[0, R_{n_{1}}\right) \quad \text { and } \quad \nu_{f}(r)=n_{k}, \quad r \in\left[R_{n_{k}}, R_{n_{k+1}}\right), \quad k \in \mathbb{N}, \\
& \mu_{f}(r)=1, \quad r \in\left[0, R_{n_{1}}\right) \quad \text { and } \quad \mu_{f}(r)=F_{n_{k}} r^{n_{k}}, \quad r \in\left[R_{n_{k}}, R_{n_{k+1}}\right), \quad k \in \mathbb{N} .
\end{aligned}
$$

Let, as above, $\Lambda_{f}=\left\{\lambda_{m}\right\}_{m=1}^{\infty}$ be the sequence of the zeroes of the function $f$ taken counting their multiplicities in the ascending order of their absolute values, see (2):

$$
0<\left|\lambda_{1}\right|=\ldots=\left|\lambda_{m_{1}}\right|<\left|\lambda_{m_{1}+1}\right|=\ldots=\left|\lambda_{m_{2}}\right|<\ldots
$$

The counting function of the zeroes $\Lambda_{f}$ of the function $f$ defined by the formula

$$
n(r)=n_{f}(r)=\max \left\{m \in \mathbb{N}:\left|\lambda_{m}\right| \leqslant r\right\},
$$

is introduced by the following identities:

$$
n(r)=0, \quad r \in\left[0,\left|\lambda_{m_{1}}\right|\right) \quad \text { and } n(r)=m_{k}, \quad r \in\left[\left|\lambda_{m_{k}}\right|,\left|\lambda_{m_{k+1}}\right|\right), \quad k \in \mathbb{N} .
$$

Comparing with (11), we see that $n(r)$ is a central index of the function

$$
\Psi(z)=1+\sum_{m=1}^{\infty} \frac{z^{m}}{\left|\lambda_{1} \cdots \lambda_{m}\right|},
$$

and hence, it possesses the same properties as $\nu_{f}(r)$.

\section{MAIN RESUlts}

The next theorem strengthens inequality (7).

Theorem 3.1. Given an entire function

$$
f(z)=\sum_{n=0}^{\infty} f_{n} z^{n} \in E_{0},
$$

let $F_{n}$ be the Hadamard-rectified coefficients of $f(z)$. Then the inequality holds:

$$
\varliminf_{n \rightarrow \infty} \sqrt[n]{F_{n}\left|\lambda_{1} \lambda_{2} \cdots \lambda_{n}\right|} \geqslant 1 .
$$


Proof. According to Jensen theorem, for each $r>0$ the identity holds:

$$
\ln \frac{r^{n(r)}}{\left|\lambda_{1} \lambda_{2} \cdots \lambda_{n(r)}\right|}=N_{f}(r)=\frac{1}{2 \pi} \int_{-\pi}^{\pi} \ln \left|f\left(r e^{i \theta}\right)\right| d \theta .
$$

For all $r>0$ and all $n \in \mathbb{N}$ this implies the inequalities:

$$
\ln \frac{r^{n}}{\left|\lambda_{1} \lambda_{2} \cdots \lambda_{n}\right|} \leqslant N_{f}(r) \leqslant \ln M_{f}(r) .
$$

Since for each $h>1$ the condition

$$
M_{f}(r)=o\left(\mu_{f}(h r)\right), \quad r \rightarrow+\infty,
$$

holds, then for sufficiently large $r$ we have:

$$
M_{f}(r) \leqslant \mu_{f}(h r), \quad r>r_{0}(h) .
$$

An obvious corollary of inequalities (13) and (14) is the estimate

$$
N_{f}(r) \leqslant \ln \mu_{f}(h r), \quad h>1, \quad r>r_{0}(h) .
$$

By this estimate we get:

$$
\begin{aligned}
& \frac{r^{n}}{\left|\lambda_{1} \lambda_{2} \cdots \lambda_{n}\right|} \leqslant \mu_{f}(h r), \quad \frac{1}{\left|\lambda_{1} \lambda_{2} \cdots \lambda_{n}\right|} \leqslant \frac{\mu_{f}(h r)}{r^{n}}, \\
& \frac{1}{\left|\lambda_{1} \lambda_{2} \cdots \lambda_{n}\right|} \leqslant h^{n} \inf _{r>r_{0}(h)} \frac{\mu_{f}(h r)}{(h r)^{n}}=h^{n} F_{n}, \quad n>n_{0}(h) .
\end{aligned}
$$

Here we have taken into account that the central index increases unboundedly as $r$ grows. The latter inequality implies the relations

$$
F_{n}\left|\lambda_{1} \lambda_{2} \cdots \lambda_{n}\right| \geqslant h^{-n}, \quad n>n_{0}(h), \quad \varliminf_{n \rightarrow \infty} \sqrt[n]{F_{n}\left|\lambda_{1} \lambda_{2} \cdots \lambda_{n}\right|} \geqslant h^{-1} .
$$

Since $h>1$ is arbitrary, we obtain needed estimate 12 . The proof is complete.

We observe that the estimate in the above proven theorem is sharp. Owing to the result by Oskolkov, see (6), this inequality becomes the identity, for instance, on the functions with logarithmically convex coefficients (then $\left|f_{n}\right|=F_{n}, n \in \mathbb{N}_{0}$ ), the zeroes of which obey condition (5).

Taking into consideration the increasing of the sequence $\left|\lambda_{n}\right|$, we obtain the statement from the Hadamard theorem [14]:

$$
\varliminf_{n \rightarrow \infty}\left|\lambda_{n}\right| \sqrt[n]{F_{n}} \geqslant 1
$$

Moreover, employing (10), we obtain

$$
\varlimsup_{n \rightarrow \infty}\left|\lambda_{n}\right| \sqrt[n]{\left|f_{n}\right|} \geqslant \varlimsup_{k \rightarrow \infty}\left|\lambda_{n_{k}}\right| \sqrt[n]{\left|f_{n_{k}}\right|}=\varlimsup_{k \rightarrow \infty}\left|\lambda_{n_{k}}\right| \sqrt[n]{k}{ }_{F_{n_{k}}} \geqslant \varliminf_{n \rightarrow \infty}\left|\lambda_{n}\right| \sqrt[n]{F_{n}} \geqslant 1
$$

that is, estimate (7) holds:

$$
\varlimsup_{n \rightarrow \infty}\left|f_{n}\right| \sqrt[n]{\left|\lambda_{n}\right|} \geqslant 1
$$

Finally, employing (12) and the fact the upper D'Alembert limit is not less than the Cauchy upper limit! we get:

$$
\varlimsup_{n \rightarrow \infty} \frac{\left|\lambda_{n}\right|}{R_{n}} \geqslant \varlimsup_{n \rightarrow \infty} \sqrt[n]{\frac{\left|\lambda_{1} \lambda_{2} \cdots \lambda_{n}\right|}{R_{1} R_{2} \cdots R_{n}}}=\varlimsup_{n \rightarrow \infty} \sqrt[n]{F_{n}\left|\lambda_{1} \lambda_{2} \cdots \lambda_{n}\right|} \geqslant 1 .
$$

\footnotetext{
${ }^{1}$ This is implied by Stolz inequality.
} 
Thus, we arrive at the following corollary.

Corollary 3.1. Given $f(z)=\sum_{n=0}^{\infty} f_{n} z^{n} \in E_{0}$, let $F_{n}$ be the Hadamard-rectified coefficients of $f(z), R_{n}=\frac{F_{n-1}}{F_{n}}$ and $\left\{\lambda_{n}\right\}$ be the sequence of all zeroes of this function taken in the ascending order of their absolute values. Then the inequality holds:

$$
\varlimsup_{n \rightarrow \infty} \frac{\left|\lambda_{n}\right|}{R_{n}} \geqslant 1 \text {. }
$$

The inequality in the above corollary is sharp, the identity is attained at some class of entire functions with lacunary Taylor series. This will be seen from the results of the previous section, in which we establish two-sided estimates of the quotients $\frac{\left|\lambda_{n}\right|}{R_{n}}$, see, for instance, Theorem 4.1 .

We are going to show that inequality (15) implies estimate (8) in work [18] cited in the introduction. Indeed, the assumption that estimate (8) fails, implies that for some $q \in(0,1)$, the inequality holds:

$$
\left|\lambda_{m}\right| \sqrt[m]{F_{m}}<q e^{\frac{\ln F_{m} R_{m}^{m}}{m}}, \quad m>m_{0}
$$

After some elementary transformations we arrive at the estimate

$$
\left|\lambda_{m}\right|<q R_{m}, \quad m>m_{0}
$$

which contradicts 15 .

Our next step is to prove a result specifying estimate (9). We first introduce the following quantities:

$$
\underline{\nu}_{f}=\varliminf_{r \rightarrow+\infty} \frac{N_{f}(r)}{n_{f}(r)}, \quad \bar{\nu}_{f}=\varlimsup_{r \rightarrow+\infty} \frac{N_{f}(r)}{n_{f}(r)}
$$

called in work [21] respectively upper and lower relative densities of the sequence $\Lambda_{f}$.

Theorem 3.2. Given an entire function

$$
f(z)=\sum_{n=0}^{\infty} f_{n} z^{n} \in E_{0},
$$

let $F_{n}$ be the Hadamard-rectified coefficients of $f(z)$, and $\underline{\nu}_{f}$ be the lower relative density of its zeroes. Then an inequality holds:

$$
\varliminf_{n \rightarrow \infty}\left|\lambda_{n}\right| \sqrt[n]{F_{n}} \geqslant e^{\underline{\nu}_{f}}
$$

Proof. We transform:

$$
\begin{aligned}
\underline{\nu}_{f} & =\varliminf_{r \rightarrow+\infty} \frac{N_{f}(r)}{n_{f}(r)}=\varliminf_{k \rightarrow \infty}\left(\inf _{r \in\left[\left|\lambda_{m_{k}}\right|,\left|\lambda_{m_{k+1}}\right|\right)} \frac{N_{f}(r)}{n_{f}(r)}\right)=\varliminf_{k \rightarrow \infty} \frac{N\left(\left|\lambda_{m_{k}}\right|\right)}{m_{k}} \\
& =\varliminf_{k \rightarrow \infty}\left(\operatorname{linf}_{m \in\left(m_{k-1}, m_{k}\right]} \frac{N\left(\left|\lambda_{m}\right|\right)}{m}\right)=\varliminf_{m \rightarrow \infty} \frac{N\left(\left|\lambda_{m}\right|\right)}{m} .
\end{aligned}
$$

Here we have employed that $\left|\lambda_{m}\right|=\left|\lambda_{m_{k}}\right|$ for $m \in\left(m_{k-1}, m_{k}\right]$. Let $M$ be the set of the indices, on which the lower limit is attained in estimate $(16)$, that is,

$$
\varliminf_{m \rightarrow \infty}\left|\lambda_{m}\right| \sqrt[m]{F_{m}}=\lim _{m \in M}\left|\lambda_{m}\right| \sqrt[m]{F_{m}}
$$

Suppose that inequality (16) fails. Then for some $q \in(0,1)$ and all $m \in M, m>m_{0}$ we have:

$$
\left|\lambda_{m}\right| \sqrt[m]{F_{m}}<q e^{\frac{N\left(\left|\lambda_{m}\right|\right)}{m}}
$$


or, taking the $m$ th power,

$$
\left|\lambda_{m}\right|^{m} F_{m}<q^{m} e^{N\left(\left|\lambda_{m}\right|\right)}=q^{m} \frac{\left|\lambda_{m}\right|^{m}}{\left|\lambda_{1}\right| \cdots\left|\lambda_{m}\right|} .
$$

After dividing by $\left|\lambda_{m}\right|^{m}$, we get:

$$
\begin{gathered}
F_{m}<\frac{q^{m}}{\left|\lambda_{1}\right| \cdots\left|\lambda_{m}\right|}, \quad F_{m}\left|\lambda_{1}\right| \cdots\left|\lambda_{m}\right|<q^{m}, \\
\underline{\lim _{m \rightarrow \infty}} \sqrt[m]{F_{m}\left|\lambda_{1} \cdots \lambda_{m}\right|} \leqslant \underline{\varliminf_{m \in \mathbb{M}}} \sqrt[m]{F_{m}\left|\lambda_{1} \cdots \lambda_{m}\right|} \leqslant q<1 .
\end{gathered}
$$

But this contradicts inequality 12 of Theorem 3.1. The proof is complete.

Estimate (16) involves lower limits. For the upper limits of the same quantities, the following result holds true.

Theorem 3.3. Given an entire function

$$
f(z)=\sum_{n=0}^{\infty} f_{n} z^{n} \in E_{0}
$$

let $F_{n}$ be the Hadamard-rectified coefficients of $f(z)$, and $\bar{\nu}_{f}$ be the upper relative density of its zeroes. Then an inequality holds:

$$
\varlimsup_{n \rightarrow \infty}\left|\lambda_{n+1}\right| \sqrt[n]{F_{n}} \geqslant e^{\bar{\nu}_{f}}
$$

Proof. We proceed as in the proof of inequality (16). In this case we have:

$$
\bar{\nu}_{f}=\varlimsup_{r \rightarrow+\infty} \frac{N_{f}(r)}{n_{f}(r)}=\varlimsup_{k \rightarrow \infty}\left(\sup _{r \in\left[\left|\lambda_{m_{k}}\right|,\left|\lambda_{m_{k+1}}\right|\right)} \frac{N_{f}(r)}{n_{f}(r)}\right)=\varlimsup_{k \rightarrow \infty} \frac{N_{f}\left(\left|\lambda_{m_{k+1}}\right|\right)}{m_{k}}=: \lim _{k \in \mathbb{K}} \frac{N_{f}\left(\left|\lambda_{m_{k+1}}\right|\right)}{m_{k}} .
$$

Here $K$ is the set of indices, on which the upper limit is attained in the latter identity. By the definition of the upper relative density, for a small $\varepsilon>0$ we find an index $k_{0}$ such that for $k>k_{0}, k \in \mathbb{K}$, the inequality holds:

$$
\frac{N_{f}\left(\left|\lambda_{m_{k+1}}\right|\right)}{m_{k}}>\bar{\nu}_{f}-\varepsilon
$$

We shall also need the relation

$$
N_{f}\left(\left|\lambda_{m_{k+1}}\right|\right)-N_{f}\left(\left|\lambda_{m_{k}}\right|\right)=\int_{\left|\lambda_{m_{k}}\right|}^{\left|\lambda_{m_{k+1}}\right|} \frac{n_{f}(t)}{t} d t=m_{k} \ln \frac{\left|\lambda_{m_{k+1}}\right|}{\left|\lambda_{m_{k}}\right|}
$$

implied by the definition of $N_{f}(r)$. Suppose that (17) fails, i.e.,

$$
\varlimsup_{n \rightarrow \infty}\left|\lambda_{n+1}\right| \sqrt[n]{F_{n}}<e^{\bar{\nu}_{f}}
$$

Then for some $q \in(0,1)$ and all sufficiently large $n>n_{0}$ we have:

$$
\left|\lambda_{n+1}\right| \sqrt[n]{F_{n}}<q e^{\bar{\nu}_{f}-\varepsilon}
$$

Letting here $n=m_{k}, k \in \mathbb{K}, k>k_{0}$, in view of (18) and (19) we obtain:

$$
\left|\lambda_{m_{k+1}}\right| \sqrt[m k]{F_{m_{k}}}<q e^{\frac{N_{f}\left(\left|\lambda_{m_{k+1}}\right|\right)}{m_{k}}}=q \frac{\left|\lambda_{m_{k+1}}\right|}{\left|\lambda_{m_{k}}\right|} e^{\frac{N_{f}\left(\left|\lambda_{m_{k}}\right|\right)}{m_{k}}}
$$

or, after cancellation and taking the $m_{k}$ th power,

$$
F_{m_{k}}<\frac{q^{m_{k}}}{\left|\lambda_{1} \lambda_{2} \cdots \lambda_{m_{k}}\right|}
$$


As in the proof of the previous theorem, this leads to a contradiction with inequality 12 . The proof is complete.

Taking into consideration the known relations between the orders and relative densities [22, Thm. 2.6.1(d)]:

$$
\underline{\nu}_{f} \leqslant \frac{1}{\rho_{f}} \leqslant \frac{1}{\lambda_{f}} \leqslant \bar{\nu}_{f}
$$

where $\rho_{f}$ and $\lambda_{f}$ are the order and the lower order of an entire function $f$, respectively, by Theorem 3.3 we obtain the following corollary.

Corollary 3.2. Let the assumptions of Theorem 3.3 be satisfied. Then the estimate

$$
\varlimsup_{n \rightarrow \infty}\left|\lambda_{n+1}\right| \sqrt[n]{F_{n}} \geqslant e^{\frac{1}{\lambda_{f}}}
$$

holds. In particular, for entire functions of zero lower order we have

$$
\varlimsup_{n \rightarrow \infty}\left|\lambda_{n+1}\right| \sqrt[n]{F_{n}}=+\infty
$$

Let us consider an example. Let $f(z)=e^{z^{\rho}}-1, \rho \in \mathbb{N}$. Then we have

$$
f(z)=\sum_{k=1}^{\infty} \frac{z^{\rho k}}{k !}
$$

and simple calculations give:

$$
R_{n}=\sqrt[\rho]{\frac{n}{\rho}}, \quad \nu(r)=\max \left\{n: R_{n} \leqslant r\right\}=\left[\rho r^{\rho}\right], \quad \ln \mu(r) \sim \ln M(r)=r^{\rho}, \quad r \rightarrow \infty .
$$

The zeroes of functions are found by the condition $z^{\rho}=2 \pi k i$, where $k \in \mathbb{Z}$. All zeroes are simple except the zero $z=0$ of multiplicity $\rho$. The circumference of the radius $\sqrt[\rho]{2 \pi|k|}$, $k \in \mathbb{Z} \backslash\{0\}$, centered at the origin contains $2 \rho$ zeroes. For non-trivial zeroes indexed by $n \in \mathbb{N}$ in the ascending order of their absolute values we write

$$
\left|\lambda_{n}\right|=\sqrt[\rho]{2 \pi\left(1+\left[\frac{n-1}{2 \rho}\right]\right)} \sim \sqrt[\rho]{\frac{\pi n}{\rho}}, \quad n \rightarrow \infty,
$$

where $[x]$ denotes the integer part of a number $x$. The above calculations lead us to the formulae

$$
\begin{aligned}
& \lim _{n \rightarrow \infty} \frac{\left|\lambda_{n}\right|}{R_{n}}=\sqrt[\rho]{\pi}, \quad \nu:=\lim _{r \rightarrow+\infty} \frac{\ln \mu(r)}{\nu(r)}=\frac{1}{\rho} \\
& \varliminf_{n \rightarrow \infty}\left|\lambda_{n}\right| \sqrt[n]{F_{n}}=\varlimsup_{n \rightarrow \infty}\left|\lambda_{n+1}\right| \sqrt[n]{F_{n}}=\lim _{m \rightarrow \infty}\left|\lambda_{\rho m}\right| \sqrt[\rho m]{F_{\rho m}}=\lim _{m \rightarrow \infty} \sqrt[\rho]{\pi m} \sqrt[\rho m]{\frac{1}{m !}}=\sqrt[\rho]{\pi e}
\end{aligned}
$$

We note that the Taylor series of the considered function is lacunary with lacunas of size $\rho$. At that, each of general formulae (15)-(17) differs from the corresponding formula in the above formula by the factor $\sqrt[\rho]{\pi}$ tending to one as $\rho$ grows unboundedly. This tendency will arise in the next section devoted to lacunary series, see Theorem 4 below. The sharpness of the above obtained results is also ensured by an example considered in [23, Thm. 3]; this function was

$$
f(z)=e^{z^{\rho}} g(z)+a,
$$

where $\rho \in \mathbb{N}, a \in \mathbb{C}$ and $g(z)$ is an entire function of the minimal type at order $\rho$. 


\section{Estimates For LACUNARY TAYLOR SERIES}

In this section we improve the methods going back to Hadamard, Borel and Valiron and we obtain asymptotic and uniform estimates relating the zeroes and Taylor coefficients of entire lacunary power series:

$$
f(z)=\sum_{n \in \mathbb{N}_{1}} f_{n} z^{n}, \quad f_{n} \neq 0, \quad n \in \mathbb{N}_{1}=\left\{n_{i}\right\} \subset \mathbb{N} .
$$

We employ the above introduced notations: $F_{n}$ are the Hadamard-rectified coefficients, $R_{n}=$ $\frac{F_{n-1}}{F_{n}}$, and $m_{k}$ are the central indices 20. We also let

$$
\alpha_{k}=\frac{R_{m_{k+1}}}{R_{m_{k}}}, \quad \gamma_{i}=n_{i}-n_{i-1}, \quad n_{i} \in \mathbb{N}_{1} .
$$

Central lacunas are lacunas adjoint with the central indices $m_{k}$ of the function $f$. Here we mean the lacunas

$$
\gamma_{k}^{\prime}=m_{k}-n_{k}^{\prime}, \quad \gamma_{k}^{\prime \prime}=n_{k}^{\prime \prime}-m_{k}, \quad \tilde{\gamma}_{k}=\min \left\{\gamma_{k}^{\prime}, \gamma_{k}^{\prime \prime}\right\}
$$

where

$$
n_{k}^{\prime}=\max \left\{n \in \mathbb{N}_{1}: n<m_{k}\right\}, \quad n_{k}^{\prime \prime}=\min \left\{n \in \mathbb{N}_{1}: n>m_{k}\right\} .
$$

Now we are in position to formulate our result.

Theorem 4.1. Let series (20) have unbounded central lacunas, namely, let the condition hold:

$$
\lim _{k \rightarrow \infty} \tilde{\gamma}_{k}=\infty
$$

Let also the following conditions on the coefficients be satisfied:

$$
\frac{R_{m_{k+1}}}{R_{m_{k}}}:=\alpha_{k} \geqslant\left(1+\frac{\ln \tilde{\gamma}_{k}}{\tilde{\gamma}_{k}}\right)^{2}, \quad k \in \mathbb{N} .
$$

Then function (20) has infinitely many zeroes $\lambda_{n}, n \in \mathbb{N}$, and an asymptotic relation holds:

$$
\left|\lambda_{n}\right| \sim R_{n}, \quad n \rightarrow \infty
$$

Proof. We fix $k \in \mathbb{N}$. For the values $r \in\left[R_{m_{k}}, R_{m_{k+1}}\right)$, as a central index of the function $f$, the quantity $\nu_{f}(r)=m_{k}$ serves, while the maximal term is $\mu_{f}(r)=\left|f_{m_{k}}\right| r^{m_{k}}$. We partition the series in 20 as follows:

$$
f(z)=\sum_{n \in \mathbb{N}_{1}} f_{n} z^{n}=\sum_{n<m_{k}, n \in \mathbb{N}_{1}} f_{n} z^{n}+f_{m_{k}} z^{m_{k}}+\sum_{n>m_{k}, n \in \mathbb{N}_{1}} f_{n} z^{n} .
$$

We choose numbers $p_{k}, q_{k}$ in the interval $(0,1)$ so that $p_{k} q_{k}>\alpha_{k}^{-1}$ and let us estimate the sums

$$
S_{1}(z):=\sum_{n<m_{k}, n \in \mathbb{N}_{1}} f_{n} z^{n}, \quad S_{2}(z):=\sum_{n>m_{k}, n \in \mathbb{N}_{1}} f_{n} z^{n}
$$


on the circumferences $|z|=r=\alpha R_{m_{k}}$, where $\alpha \in\left[\frac{1}{p_{k}}, q_{k} \alpha_{k}\right)$. We have

$$
\begin{aligned}
\frac{\left|S_{1}(z)\right|}{\mu_{f}(r)} & \leqslant \sum_{n<m_{k}, n \in \mathbb{N}_{1}}\left|\frac{f_{n}}{f_{m_{k}}}\right| \frac{1}{r^{m_{k}-n}} \leqslant \sum_{n<m_{k}, n \in \mathbb{N}_{1}} \frac{F_{n}}{F_{m_{k}}} \frac{1}{r^{m_{k}-n}} \\
& \leqslant \sum_{n<m_{k}, n \in \mathbb{N}_{1}} \frac{R_{n+1} \cdots R_{m_{k}}}{r^{m_{k}-n}} \leqslant \sum_{n<m_{k}, n \in \mathbb{N}_{1}}\left(\frac{R_{m_{k}}}{r}\right)^{m_{k}-n} \\
& =\sum_{n<m_{k}, n \in \mathbb{N}_{1}}\left(\frac{1}{\alpha}\right)^{m_{k}-n} \leqslant\left(\frac{1}{\alpha}\right)^{m_{k}-n_{k}^{\prime}} \sum_{i=0}^{\infty} \alpha^{-i} \\
& =\frac{\left(\frac{1}{\alpha}\right)^{m_{k}-n_{k}^{\prime}}}{1-\alpha^{-1}} \leqslant \frac{p_{k}^{m_{k}-n_{k}^{\prime}}}{1-p_{k}} .
\end{aligned}
$$

Thus, estimate

$$
\left|S_{1}(z)\right| \leqslant \mu_{f}(r) \frac{p_{k}^{\gamma_{k}^{\prime}}}{1-p_{k}}, \quad|z|=r=\alpha R_{m_{k}} \in\left[p_{k}^{-1} R_{m_{k}}, R_{m_{k+1}}\right)
$$

is true. For the same $z \in \mathbb{C}:|z|=r=\alpha R_{m_{k}}$ we have:

$$
\begin{aligned}
& \left|\frac{f_{n}}{f_{m_{k}}} z^{n-m_{k}}\right| \leqslant \frac{F_{n}}{F_{m_{k}}} r^{n-m_{k}}=\frac{r^{n-m_{k}}}{R_{m_{k+1}} \cdots R_{n}} \leqslant\left(\frac{r}{R_{m_{k+1}}}\right)^{n-m_{k}}=\left(\frac{\alpha}{\alpha_{k}}\right)^{n-m_{k}}, \\
& \frac{\left|S_{2}(z)\right|}{\mu_{f}(r)} \leqslant \sum_{n>m_{k}, n \in \mathbb{N}_{1}}\left|\frac{f_{n}}{f_{m_{k}}} z^{n-m_{k}}\right| \leqslant \sum_{n>m_{k}, n \in \mathbb{N}_{1}}\left(\frac{\alpha}{\alpha_{k}}\right)^{n-m_{k}} \\
& \leqslant\left(\frac{\alpha}{\alpha_{k}}\right)^{n_{k}^{\prime \prime}-m_{k}} \sum_{i=0}^{\infty}\left(\frac{\alpha}{\alpha_{k}}\right)^{i}=\frac{\left(\frac{\alpha}{\alpha_{k}}\right)^{n_{k}^{\prime \prime}-m_{k}}}{1-\frac{\alpha}{\alpha_{k}}} \leqslant \frac{q_{k}^{n_{k}^{\prime \prime}-m_{k}}}{1-q_{k}} .
\end{aligned}
$$

Thus, the estimate holds

$$
\left|S_{2}(z)\right| \leqslant \mu_{f}(r) \frac{q_{k}^{\gamma_{k}^{\prime \prime}}}{1-q_{k}}, \quad|z|=r=\alpha R_{m_{k}} \in\left[R_{m_{k}}, q_{k} R_{m_{k+1}}\right) .
$$

Combining estimates (24) and (25), we conclude

$$
\left|S_{1}(z)\right|+\left|S_{2}(z)\right| \leqslant \mu_{f}(r) T\left(p_{k}, q_{k}\right), \quad|z|=r \in\left[p_{k}^{-1} R_{m_{k}}, q_{k} R_{m_{k+1}}\right),
$$

where

$$
T\left(p_{k}, q_{k}\right)=\frac{p_{k}^{\gamma_{k}^{\prime}}}{1-p_{k}}+\frac{q_{k}^{\gamma_{k}^{\prime \prime}}}{1-q_{k}} .
$$

Making estimates more rough and assuming for simplicity that $p_{k} \leqslant q_{k}, k \in \mathbb{N}$, we hence obtain

$$
T\left(p_{k}, q_{k}\right) \leqslant 2 \frac{q_{k}^{\tilde{\gamma}_{k}}}{1-q_{k}} .
$$

Let us show that it is possible to choose $q_{k}$ to satisfy the conditions

$$
q_{k}^{-2} \leqslant \alpha_{k}, \quad \frac{q_{k}^{\tilde{\gamma}_{k}}}{1-q_{k}} \rightarrow 0, \quad k \rightarrow \infty
$$

In order to do this, we denote $\varepsilon_{k}=\frac{\ln \tilde{\gamma}_{k}}{\tilde{\gamma}_{k}}$ and we let $q_{k}^{-1}=1+\varepsilon_{k}$. Indeed,

$$
q_{k}^{-2}=\left(1+\varepsilon_{k}\right)^{2} \leqslant \alpha_{k}
$$


and the first condition in (27) is satisfied. Since $\varepsilon_{k} \rightarrow 0$ as $k \rightarrow \infty$, then $q_{k} \rightarrow 1$ and we get:

$$
\begin{aligned}
\frac{q_{k}^{\tilde{\gamma}_{k}}}{1-q_{k}} & =\frac{1}{q_{k}} \frac{\left(1 / q_{k}\right)^{-\tilde{\gamma}_{k}}}{1 / q_{k}-1} \sim \frac{1}{\varepsilon_{k}}\left(1+\varepsilon_{k}\right)^{-\tilde{\gamma}_{k}}=\frac{1}{\varepsilon_{k}}\left[\left(1+\varepsilon_{k}\right)^{\frac{1}{\varepsilon_{k}}+1}\right] \frac{\frac{-\tilde{\gamma}_{k}}{\varepsilon_{k}+1}}{\varepsilon_{k}} \\
& <\frac{1}{\varepsilon_{k}} e^{\frac{-\tilde{\gamma}_{k}}{\frac{1}{\varepsilon_{k}}+1}}<\frac{2}{\varepsilon_{k}} e^{-\tilde{\gamma_{k}} \varepsilon_{k}}=\frac{2 \tilde{\gamma}_{k}}{\ln \tilde{\gamma}_{k}} e^{-\frac{\tilde{\gamma}_{k} \ln \tilde{\gamma}_{k}}{\tilde{\gamma}_{k}}}=\frac{2}{\ln \tilde{\gamma}_{k}} \rightarrow 0, \quad k \rightarrow \infty,
\end{aligned}
$$

and we see that the second condition in (27) is satisfied as well. Taking into considerations estimates (26) and (27), we conclude

$$
\left|S_{1}(z)\right|+\left|S_{2}(z)\right|<\mu_{f}(r), \quad|z|=r \in\left[p_{k}^{-1} R_{m_{k}}, q_{k} R_{m_{k+1}}\right), \quad k>k_{0} .
$$

According Rouché theorem, as $k>k_{0}$, in the circle $|z|<p_{k}^{-1} R_{m_{k}}$ the function $f(z)=$ $S_{1}(z)+S_{2}(z)+f_{m_{k}} z^{m_{k}}$ has the same amount of zeroes as the function $f_{m_{k}} z^{m_{k}}$ does, that is, $m_{k}$ zeroes, while in the circle $|z|<q_{k-1}^{-1} R_{m_{k}}$ it has $m_{k-1}$ zeroes. This implies that for sufficiently large $k$, the annulus

$$
q_{k-1}^{-1} R_{m_{k}}<|z|<p_{k}^{-1} R_{m_{k}}, \quad k>k_{0},
$$

contains $m_{k}-m_{k-1}$ zeroes of the function $f(z)$. Therefore, for the zeroes $\lambda_{n}$ of this function with the indices $n \in\left(m_{k-1}, m_{k}\right]$, the inequalities hold:

$$
q_{k-1}^{-1}<\frac{\left|\lambda_{n}\right|}{R_{m_{k}}}<p_{k}^{-1}, \quad n \in\left(m_{k-1}, m_{k}\right], \quad k>k_{0} .
$$

However, for such indices $n$ we have $R_{n}=R_{m_{k}}$ and we finally obtain:

$$
q_{k-1}^{-1}<\frac{\left|\lambda_{n}\right|}{R_{n}}<p_{k}^{-1}, \quad n \in\left(m_{k-1}, m_{k}\right], \quad k>k_{0} .
$$

Hence, we conclude that

$$
1=\lim _{k \rightarrow \infty} q_{k-1}^{-1} \leqslant \varliminf_{n \rightarrow \infty} \frac{\left|\lambda_{n}\right|}{R_{n}} \leqslant \varlimsup_{n \rightarrow \infty} \frac{\left|\lambda_{n}\right|}{R_{n}} \leqslant \lim _{k \rightarrow \infty} p_{k}^{-1}=1,
$$

and relation $(23)$ holds true. The proof is complete.

We note that asymptotics (23) proves the sharpness of estimate (15) in Corollary 3.1 from Theorem 3.1. Now we have two remarks.

Remark 4.1. The quantity of the initial term $f_{0}$ in series (20) makes no influence on the asymptotics of the zeroes of the function $f$ and this is why the statement of Theorem 4.1 is true not only for the zeroes, but also for all a-points of the function $f$, that is, for the zeroes of the function $f-a, a \in \mathbb{C}$.

Remark 4.2. Condition (21) of Theorem 4.1 is obviously satisfied if all but not only central lacunas in series (20) are unbounded, that is, if

$$
\lim _{i \rightarrow \infty} \gamma_{i}=\lim _{i \rightarrow \infty}\left(n_{i}-n_{i-1}\right)=\infty
$$

The condition in the latter remark is easier for checking since it does not require finding the values of the central index of the series. Such checking is even not needed if the sequence $\left\{\left|f_{n}\right|\right\}$, where $n \in \mathbb{N}_{1}$, is strictly logarithmically convex since then $\mathbb{N}_{1}=\left\{m_{k}\right\}$, that is, this set consists of all central indices of series (20). In this case we can specify the location of zeroes. 
Theorem 4.2. Let the assumptions of Theorem 4.1 be satisfied and moreover, the sequence $\left\{\left|f_{n}\right|\right\}$, where $n \in \mathbb{N}_{1}$, is strictly logarithmically convex. Then the zeroes $\left\{\lambda_{n}\right\}$ of function (20) satisfies the asymptotic formula:

$$
\lambda_{n}=\left(-\frac{f_{m_{k-1}}}{f_{m_{k}}}\right)^{\frac{1}{m_{k}-m_{k-1}}}(1+o(1)), \quad n \in\left(m_{k-1}, m_{k}\right], \quad k \rightarrow \infty,
$$

where the exponent is chosen depending on $n$.

Proof. Indeed, in the considered case the set over which series (20) is summed up consists of the values of the central indices: $\mathbb{N}_{1}=\left\{m_{k}\right\}$. This is why the identities hold:

$$
\left|f_{m_{k}}\right|=F_{m_{k}}, \quad \gamma_{k}=m_{k}-m_{k-1}, \quad R_{m_{k}}=\left|\frac{f_{m_{k-1}}}{f_{m_{k}}}\right|^{\frac{1}{\gamma_{k}}}, \quad k \in \mathbb{N} .
$$

We represent the series in 20 as

$$
\begin{aligned}
\sum_{n \in \mathbb{N}_{1}} f_{n} z^{n} & =\sum_{n<m_{k-1}, n \in \mathbb{N}_{1}} f_{n} z^{n}+f_{m_{k-1}} z^{m_{k-1}}+f_{m_{k}} z^{m_{k}}+\sum_{n>m_{k}, n \in \mathbb{N}_{1}} f_{n} z^{n} \\
& =: S_{1}^{\prime}(z)+f_{m_{k-1}} z^{m_{k-1}}+f_{m_{k}} z^{m_{k}}+S_{2}(z) .
\end{aligned}
$$

It is obvious that the sum $S_{1}^{\prime}$ admits same estimate (24) as $S_{1}$ does; the sum $S_{2}$ and its estimate (25) remain the same. This is why estimate (26) also remains true:

$$
\left|S_{1}^{\prime}(z)\right|+\left|S_{2}(z)\right| \leqslant \mu_{f}(r) T\left(p_{k}, q_{k}\right), \quad|z|=r \in\left[p_{k}^{-1} R_{m_{k}}, q_{k} R_{m_{k+1}}\right) .
$$

Taking into consideration this estimate, for $z$, satisfying the inequality

$$
\left|f_{m_{k-1}} z^{m_{k-1}}+f_{m_{k}} z^{m_{k}}\right| \geqslant\left|f_{m_{k}} z^{m_{k}}\right|
$$

we obtain:

$$
\begin{aligned}
|f(z)| & =\left|S_{1}^{\prime}(z)+f_{m_{k-1}} z^{m_{k-1}}+f_{m_{k}} z^{m_{k}}+S_{2}(z)\right| \\
& \geqslant\left|f_{m_{k-1}} z^{m_{k-1}}+f_{m_{k}} z^{m_{k}}\right|-\left(\left|S_{1}^{\prime}(z)\right|+\left|S_{2}(z)\right|\right)>\mu_{f}(r)\left(1-T\left(p_{k}, q_{k}\right)\right)>0
\end{aligned}
$$

as $k>k_{0}$. Therefore, the zeroes of the function $f$ lie in the intersection of annuli (29) and the sets

$$
\left|\frac{f_{m_{k-1}}}{f_{m_{k}}}+z^{m_{k}-m_{k-1}}\right|<r^{m_{k}-m_{k-1}}, \quad|z|=r, \quad k>k_{0} .
$$

Each such set is the pre-image of the half-plane $\left|\frac{f_{m_{k-1}}}{f_{m_{k}}}+t\right|<|t|$ under the mapping by the function $t=z^{m_{k}-m_{k-1}}=z^{\gamma_{k}}$. Denoting

$$
\varphi_{k}=\arg \left(-\frac{f_{m_{k-1}}}{f_{m_{k}}}\right)
$$

in this half-plane we have $\arg t \in\left(\varphi_{k}-\pi / 2, \varphi_{k}+\pi / 2\right)$. Returning back to the plane of the variable $z$, for the zeroes $\lambda_{n}$ we obtain, that for some integer $s_{n} \in\left[0, \gamma_{k}\right)$ we have:

$$
\arg \lambda_{n} \in\left(\frac{\varphi_{k}+2 \pi s_{n}}{\gamma_{k}}-\frac{\pi}{2 \gamma_{k}}, \frac{\varphi_{k}+2 \pi s_{n}}{\gamma_{k}}+\frac{\pi}{2 \gamma_{k}}\right) \text {. }
$$

Here for each $n$ we choose some corresponding number $s_{n} \in\left[0, \gamma_{k}\right)$ fixing the value of the root. This completes the proof since $\frac{\pi}{2 \gamma_{k}} \rightarrow 0, k \rightarrow \infty$.

We note that formula (30) is an analogue of Valiron formula (4) obtained for series (1) with no lacunas. 
Remark 4.3. It is possible that condition (22) is not necessary for the validity of Theorem 4.1. However, the author has not succeeded to exclude it from the formulation. Nevertheless, this condition can be weakened a little by assuming that for some $a \in(0,1)$, the inequality

$$
\frac{R_{m_{k+1}}}{R_{m_{k}}}=\alpha_{k} \geqslant\left(1+\frac{\ln \left(\tilde{\gamma}_{k} / \ln ^{a} \tilde{\gamma}_{k}\right)}{\tilde{\gamma}_{k}}\right)^{2}, \quad k \in \mathbb{N}, \quad k>k_{0},
$$

holds.

Remark 4.4. Analysing the proof of Theorem 4.1, we see that if condition (22) and

$$
\varlimsup_{k \rightarrow \infty} \min \left\{\tilde{\gamma}_{k}, \tilde{\gamma}_{k}-1\right\}=+\infty
$$

are satisfied, where in the latter condition the upper limit is attained at the set of the indices $k \in \mathbb{K} \subset \mathbb{N}$, then asymptotics (23) remains true on the set $\mathbb{K}$ :

$$
\left|\lambda_{n}\right| \sim R_{n}, \quad n \in\left(m_{k}, m_{k+1}\right], \quad \mathbb{K} \ni k \rightarrow \infty .
$$

Now we consider the case when instead of (21), the lacunas of series (20), more precisely, its central lacunas, satisfy the condition

$$
\varliminf_{k \rightarrow \infty} \tilde{\gamma_{k}}=\rho<+\infty .
$$

It is obvious that $\rho \in \mathbb{N}$. We introduce the function

$$
\varphi_{\rho}(\tau)=\varphi(\tau)=\frac{\tau^{\rho}}{1-\tau}, \quad \tau \in[0,1)
$$

and by $\tau_{\rho}$ we denote the root of the equation $\varphi(\tau)=1 / 2$, that is, the root of the equation

$$
2 \tau^{\rho}+\tau-1=0
$$

in $(0,1)$. It is easy to confirm that

$$
\tau_{1}=\frac{1}{3}, \quad \tau_{2}=\frac{1}{2}, \quad \tau_{3}=0.5897 \ldots,
$$

and in general, $\tau_{\rho} \nearrow 1$ as $\rho \in \mathbb{N}$ grows unboundedly.

Theorem 4.3. Let the lacunas of series (20) satisfy condition (31) and its coefficients obey the condition

$$
\frac{R_{m_{k+1}}}{R_{m_{k}}} \geqslant \beta>\tau_{\rho}^{-2}, \quad k \in \mathbb{N}, \quad k>k_{0},
$$

in which $\tau_{\rho}$ is the root of equation (32). Then the zeroes $\lambda_{n}$ of function (20) satisfy inequalities

$$
x_{\rho} \leqslant \frac{\left|\lambda_{n}\right|}{R_{n}} \leqslant \frac{1}{x_{\rho}}, \quad n \geqslant n_{0},
$$

where $x_{\rho}$ is the largest root of the equation

$$
\varphi(x)+\varphi\left(\frac{1}{x \beta}\right)=\frac{x^{\rho}}{1-x}+\frac{(\beta x)^{1-\rho}}{\beta x-1}=1 .
$$

Proof. We denote by $G$ a domain bounded by the curves defined by the equations $y x=1 / \beta$ and $\varphi(x)+\varphi(y)=1, x \in(0,1)$. Condition (33) ensures that this domain is non-empty. We shall employ the estimates obtained in the proof of Theorem 4.1 letting there $p_{k}=p, q_{k}=q$ with constants $p, q$ obeying the condition $\frac{1}{p q}<\beta$. Since in the considered case $\tilde{\gamma}_{k} \geqslant \rho$, estimate 26 becomes

$$
\left|S_{1}(z)\right|+\left|S_{2}(z)\right| \leqslant \mu_{f}(r) T(p, q), \quad|z|=r \in\left[p^{-1} R_{m_{k}}, q R_{m_{k+1}}\right),
$$


where $T(p, q)=\frac{p^{\rho}}{1-p}+\frac{q^{\rho}}{1-q}$. Estimate 28$)$ also remains true and casts into the form

$$
\left|S_{1}(z)\right|+\left|S_{2}(z)\right|<\mu_{f}(r), \quad|z|=r \in\left[p^{-1} R_{m_{k}}, q R_{m_{k+1}}\right), \quad k>k_{0},
$$

once we suppose that $(p, q) \in G$, i.e., once the condition

$$
T(p, q)=\frac{p^{\rho}}{1-p}+\frac{q^{\rho}}{1-q}=\varphi(p)+\varphi(q)<1
$$

is satisfied. As above, basing on the Rouché theorem, we obtain the inequalities:

$$
q<\frac{\left|\lambda_{n}\right|}{R_{n}}<p^{-1}, \quad n \in\left(m_{k-1}, m_{k}\right], \quad k>k_{0} .
$$

To complete the proof, it remains to approach by the point $(p, q) \in G$ the intersections of the curves enveloping the domain $G$. The proof is complete.

Since the domain $G$ is symmetric with respect to the bisectrix of the first coordinate quarter, the inequality $\tau_{\rho}<x_{\rho}$ holds. This allows us to formulate a result not requiring finding the root of equation (34).

Corollary 4.1. Let the assumptions of Theorem 4.3 hold. Then the zeroes $\lambda_{n}$ of function (20) satisfy inequalities

$$
\tau_{\rho}<\frac{\left|\lambda_{n}\right|}{R_{n}}<\frac{1}{\tau_{\rho}}, \quad n \geqslant n_{0},
$$

where $\tau_{\rho} \in(0,1)$ is the root of equation $2 \tau^{\rho}+\tau-1=0$.

In particular, for even (odd) entire functions with logarithmically convex Taylor coefficients satisfying, for sufficiently large $k \in \mathbb{N}$, the condition

$$
\frac{R_{2 k+2}}{R_{2 k}} \geqslant \beta>4, \quad\left(\frac{R_{2 k+1}}{R_{2 k-1}} \geqslant \beta>4\right)
$$

we can state a two-sided estimate

$$
\frac{1}{2}<\frac{\left|\lambda_{n}\right|}{R_{n}}<2, \quad n \geqslant n_{0}
$$

We note that in the case $\rho=1$, for instance, as the lacunas in series (20) are absent, by Theorem 4.3 we obtain the result by Hadamard [13] improved then by Valiron [6], who weakened the restriction $\beta>9$ in condition $(33)$ to the condition $\beta>4.8$. In this, as for series (20) with unbounded lacunas, Theorem 4.3 can be specified by providing the angles in the complex plane free of the zeroes of the function $f(z)$. For instance, if coefficients of series (20) are positive and logarithmically convex, then under the assumptions of Theorem 4.3 all zeroes $f(z)$ are located in the left half-plane. Some specifications of Theorem 4.3 obtained by other methods for polynomials and entire functions satisfying the condition $\frac{R_{m_{k+1}}}{R_{m_{k}}} \geqslant \beta$ for various $\beta$ can be found in work [24]. However, one fails to achieve here a desired sharpness and this problem still waits for being resolved.

\section{BIBLIOGRAPHY}

1. J. Hadamard. Sur la croissance des fonctions entières // Bull. Soc. math. 24, 186-187 (1896).

2. E. Borel. Leçons sur les fonctions entières. Gauthier-Villars, Paris (1921).

3. E. Lindelöf. Mémoire sur la théorie des fonctions entières de genre fini // Acta Societatis Scientiarum Fennicæ. XXXI:1 (1903).

4. E. Lindelöf. Sur la détermination de la croissance des fonctions entières définies par un développement de Taylor // Bull. Sci. Math. Deuxieme Serie. XXVII, 213-232 (1903). 
5. A. Pringsheim. Elementare Theorie der ganzen transzendenten Funktionen von endlicher Ordnung // Mathematische Annalen. 58, 257-342 (1904).

6. G. Valiron. Sur les fonctions entières d'ordre nul et d'ordre fini et en particulier les fonctions à correspondance règulière // Ann. Faculté des Sci. Toulouse. 3e série. 5, 117-257 (1913).

7. G. Valiron. Lectures on the general Theory of integral functions. Private, Toulouse (1923).

8. V.A. Oskolkov. On some questions in the theory of entire functions // Matem. Sborn. 184:1, 129-148 (1993). [Russ. Acad. Sci. Sb. Math. 78:1, 113-129 (1994).]

9. M.N. Sheremeta. Connection between the growth of the maximum of the modulus of an entire function and the moduli of the coefficients of its power series expansion // Izv. VUZov. Matem. 2, 100-108 (1967). (in Russian).

10. G. Valiron. Fonctions entières et mèromorphes d'une varable. Mémorial des sciences mathématiques. Fascicule 2. Gauthier-Villars, Paris (1925).

11. M.E. Iaggi. Relations entre les zero et les coefficients d'une fonction entière // Nouvelles Annales de Mathématiques. Série 4. 1, 16-19 (1901).

12. M.E. Iaggi. Sur les zéros des fonctions entières // Nouvelles Annales de Mathématiques. Série 4. 2, 218-226 (1902).

13. J. Hadamard. Sur les fonctions entières // C.R. Acad. Science Fr. Séance du 29 decembre. 13091311 (1902).

14. J. Hadamard. Étude sur les propriétées des fonctions entières et en parliculièr, une fonction, etudié par Riemann. Selecta. Gauthier-Villars, Paris (1935).

15. E. Borel. Sur les zeros des fonctions entières // Acta Math. 20, 357-396 (1897).

16. V.A. Oskolkov. Properties of functions defined by values of their linear functionals. Habilitation thesis. Moscow State Univ., Moscow (1994). (in Russian).

17. I.V. Pel'chars'ka, M.M. Sheremeta. On the value distribution and coefficients of the power expansion of an entire function // Dopov. Nats. Akad. Nauk Ukr. Mat. Pryr. Tekh. Nauky. 5, 21-25 (2005). (in Ukrainian).

18. I.V. Andrusyak. Zeros and coefficients of analytic functions // Visn. Derzh. Univ. L'viv. Politekh. 625, 43-47 (2008).

19. I.V. Andrusyak, P.V. Filevych. Coefficients of power expansion and a-points of entire function // Visn. Derzh. Univ. L'viv. Politekh. 804, 70-74 (2014).

20. A. Ostrovski. Sur les modules des zeros des fonctions entières // C.R. Acad. Sci. 206, 1541 (1938).

21. G.G. Braichev. Exact relationships between certain characteristics of growth for complex sequences // Ufimskij Matem. Zhurn. 5:4, 17-30 (2013). [Ufa Math. J. 5:4, 16-29 (2013).]

22. N.H. Bingham, C.M. Goldie, J.L. Teugels. Regular variation. Cambridge University Press. Cambridge (1989).

23. I.V. Andrusyak, P.V. Filevych. Coefficients of power expansion and a-points of an entire function with Borel exceptional value // Ukrain. Matem. Zhurn. 68:2, 147-155 (2016). [Ukrainian Math. J. 68:2, 159-170 (2016).]

24. D.M. Simeunović. Sur la répartition des zéros d'une class de polynômes // Publication de l'institut Mathématique, Nouvelle série. 28(42), 187-194 (1980).

Georgii Genrikhovich Braichev

Moscow State Pedagogical University,

Malaya Pirogovskaya str. 1, bld. 1,

119991, Moscow, Russia

E-mail: braichev@mail.ru 